Available online on 15.01.2018 at http://jddtonline.info
Journal of Drug Delivery and Therapeutics
Open Access to Pharmaceutical and Medical Research
(2011-18, publisher and licensee JDDT, This is an Open Access article which permits unrestricted non-
commercial use, provided the original work is properly cited

\title{
POLYCAPROLACTONE AS DRUG CARRIER FOR AN ANTIFUNGAL AGENT
}

\author{
Kassab Rima, Moussa Dima, Yammine Paolo* \\ Department of Chemistry, Faculty of Sciences, University of Balamand, Tripoli, Lebanon
}

\begin{abstract}
One of the most suitable and most used biodegradable polymers in particulate drug delivery is Polycaprolactone. This could be attributed to its several important characteristics including its low degradation rate, high permeability and low toxicity. In this study, Polycaprolactone microspheres loaded with the antifungal agent Amphotericin B were synthesized by means of the solvent evaporation method. The prepared microspheres had homogeneous particle sizes between 110 and $125 \mu \mathrm{m}$. they also showed a porous structure and a spherical profile. Satisfactory drug encapsulation percentages and drug loading values were obtained. They varied from 28 to $61 \%$ and 0.22 to $1.13 \%$ respectively. These observed results could be related to the properties of both drug and polymer such as hydrophobicity and solubility in organic solvents. In addition, the low degradation activity of the polymer Polycaprolactone had a direct impact on the release rate of Amphotericin B, yielding prolonged and slow release profiles for most of the formulations. Finally, Infrared study revealed that the method used for encapsulation of Amphotericin B with Polycaprolactone did not have any negative effect on the integrity and stability of the drug.
\end{abstract}

Key Words: drug delivery, solvent evaporation, Polycaprolactone, Amphotericin B, In vitro release

Article Info: Received 11 Nov, 2017; Review Completed 11 Jan, 2018; Accepted 13 Jan, 2018; Available online 15 Jan, 2018

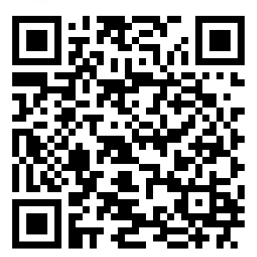

\section{Cite this article as:}

Rima K, Dima M, Paolo Y, Polycaprolactone as drug carrier for an antifungal agent, Journal of Drug Delivery and Therapeutics. 2018; 8(1):81-85

DOI: $\underline{\text { http://dx.doi.org/10.22270/jddt.v8i1.1555 }}$

*Address for Correspondence

Dr. Paolo Yammine, PhD, Associate Professor, Department of Chemistry, Faculty of Sciences, University of Balamand, Tripoli, Lebanon, Phone: 00961-6-931 952 Ext: 3841, P.O. Box: 100 Tripoli, Lebanon. E-mail: paolo.yammine@balamand.edu.lb

\section{INTRODUCTION}

Drug Delivery Systems using biodegradable polymeric particles have become one of the most important topics in the pharmaceutical and medical work. They present many advantages including sustained delivery of drugs, their localized delivery, and their stabilization in order to avoid their major side effects. Biodegradable and biocompatible polyesters such as Polylactic acid (PLA), poly(lactic-co-glycolic acid) (PLGA), polycaprolactone and their copolymers are considered essential materials for successful and efficient drug delivery systems ${ }^{1-4}$.

Polycaprolactone (PCL) is very suitable for controlled delivery due to its high permeability to a wide range of drugs, non-toxicity, and low degradation rate. PCL is a hydrophobic and semi-crystalline polymer $^{1-5}$. Its good solubility in many solvents and its exceptional blendcompatibility has enabled it to be used extensively in several medical applications. This has a positive effect on the drug release rate ${ }^{6,7}$. These advantages have made it widely used in preparation of polymeric microparticles and nanoparticles, scaffolds and tissues ${ }^{1-}$ 6

Degradation of PCL in comparison to the other polyesters is very slow, which is mainly needed for long-term delivery extending over a period of more than one year. In addition, the non-toxic products from its biodegradation also contribute in being favorable in delivery vehicles ${ }^{8,} 9$. Several drugs or biological molecules have been encapsulated into PCL microspheres or nanospheres such as Nifedipine, Indomethacin, Taxol, antigens, hormones, steroids, etc. $5-10$

In previous studies, PCL nanospheres loaded with the antifungal agent Amphotericin B (AmB) were prepared 
using the solvent displacement method ${ }^{11}$. In this project, AmB will be encapsulated into PCL microspheres by means of oil-in-water solvent evaporation technique. Preparation of microspheres with this technique is very convenient in yielding particles with a large surface area to volume ratio. This makes them very efficient in drug delivery and targeting, mainly in cases where extended and prolonged release is required ${ }^{12,13}$.

\section{MATERIALS AND METHODS}

Chemicals used: The polymer, PCL (average MW 80,000), AmB, the surfactant Tween 80, and Phosphate Buffered Saline (PBS) (0.2 M, pH 7.4) are purchased from Sigma-Aldrich, Chemie, Germany. The solvents dichloromethane (DCM) and Methanol $(\mathrm{MeOH})$ are of analytical grade.

Formulation of Microspheres: o/w emulsion solvent evaporation technique is used for the formulation of AmB-loaded PCL microspheres. It consists of mixing the organic polymeric phase with an aqueous phase. Specific drug mass is dissolved in 14:6 ml DCM/MeOH containing $500 \mathrm{mg}$ polymer, forming the organic phase. This mixture was added into $250 \mathrm{ml}$ aqueous phase containing $1 \% \mathrm{w} / \mathrm{v}$ Tween 80 . The whole combination is stirred for 6 hours at $1400 \mathrm{rpm}$ over a mechanical stirrer (MSP-1 Digital Overhead Stirrer, Jeiotech, Korea). The resulting solution is filtered to collect the obtained microspheres. These are then washed with distilled water and $\mathrm{MeOH}$, and oven dried.

\section{Characterization:}

1. Particle size and morphology: A Laser Diffraction Granulometer (LA950V2, Horiba Ltd., France) is used to determine the size of microspheres. A quantity of microspheres is suspended in water, with Tween 80 used as dispersant. The average particle size is measured in micrometers.

The morphological characteristics of microspheres are examined by Scanning Electron Microscopy (SEM) (LYRA3 XMU, TESCAN, Czech Republic). Microspheres are fixed to a carbon conductive tape. A coating of $10 \mathrm{~nm}$ of Platinum is applied using a sputter coater.

2. Drug Encapsulation (\%DE) and Drug Loading (\%DL): Microspheres are dissolved in 7/3 ml $\mathrm{DCM} / \mathrm{MeOH}$ and the drug content of each formulation is measured using a UV/Vis Spectrophotometer (Microplate Spectrophotometer, Epoch Biotek, USA) at $409 \mathrm{~nm}$. \%DE and \%DL are calculated as follows:

$$
\begin{gathered}
\% \mathrm{DE}=\frac{\text { mass of drug encapsulated }}{\text { mass of drug introduced }} * 100 \\
\% \mathrm{DL}=\frac{\text { mass of drug in microspheres }}{\text { mass of microspheres }} * 100
\end{gathered}
$$

3. In vitro drug release study: The In vitro release study is carried out in PBS solution $(0.2 \mathrm{M}, \mathrm{pH}$ 7.4). $25 \mathrm{mg}$ microspheres are introduced in small vials containing $25 \mathrm{ml}$ PBS, used as release medium, and maintained at $37^{\circ} \mathrm{C}$. At specific time intervals, $5 \mathrm{ml}$ of the release medium is withdrawn and replaced with fresh solution. It is then evaluated for its drug content at $409 \mathrm{~nm}$.

4. $\quad$ Fourier Transform-Infrared study (FT-IR): FT-IR spectra of AmB, PCL blank microspheres and AmB-loaded microspheres are determined using a FTIR spectrometer (Frontier, Perkin Elmer, USA) in order to investigate the possible chemical interactions between the drugs and the polymer matrix.

\section{RESULTS AND DISCUSSION}

The solvent evaporation is one of the most common and successful methods for drug encapsulation. It presents many positive returns including the ease of its application and the reproducibility of its results. Also, a major factor for its effectiveness is in obtaining good encapsulation values for both hydrophobic as well as hydrophilic drugs ${ }^{14}$.

\section{Particle Size and Morphology:}

AmB-loaded PCL Microspheres were homogeneous in size ranging between $110 \mu \mathrm{m}$ and $125 \mu \mathrm{m}$ (Table 1). As for the surface morphology of microspheres after examination by SEM, Figure 1 shows that they exhibited a spherical profile with a smooth and porous structure.

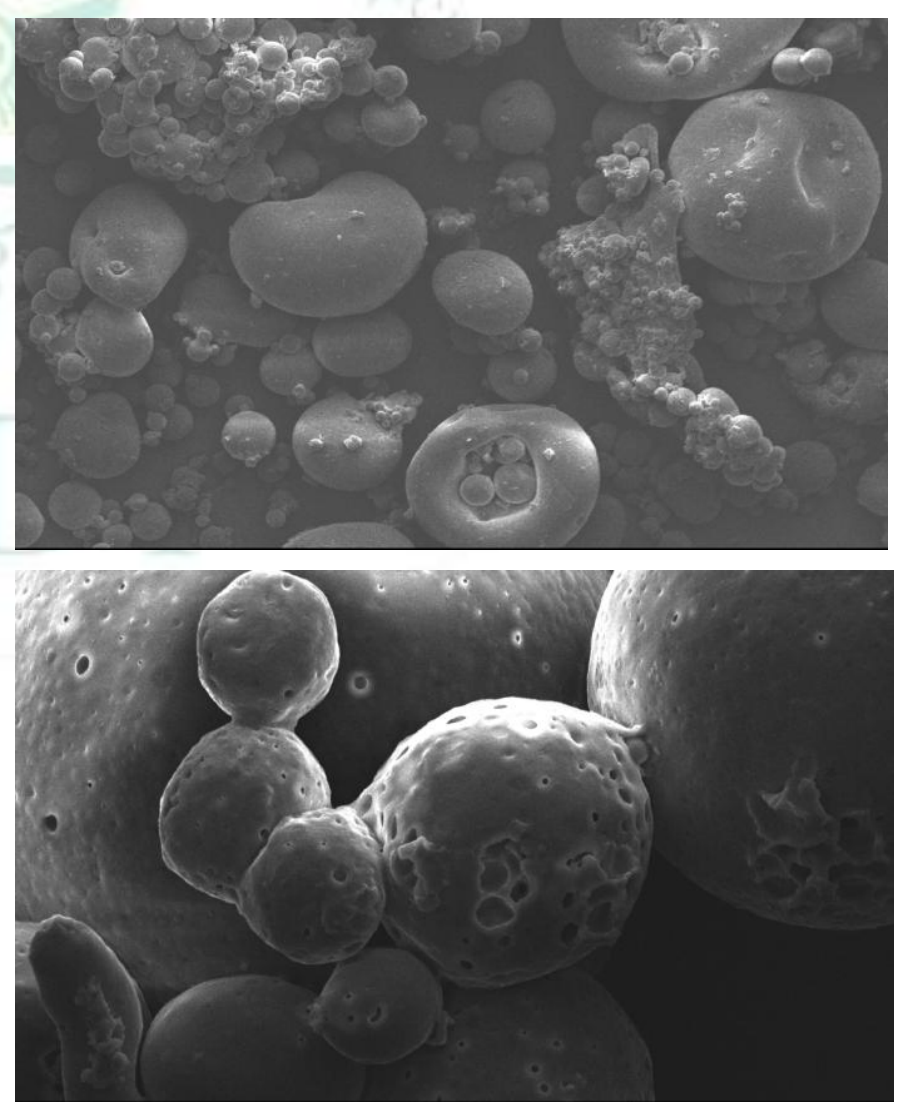

\section{Figure 1: SEM photograph of the prepared microspheres at (a) $\times 100$ and (b) $\times 1000$ magnifications}

\section{Drug Encapsulation (\%DE) and Drug Loading (\%DL):}

Good values for drug encapsulation and drug loading were obtained. Since AmB is hydrophobic in character, 
it tends to remain in the organic polymer phase, leading to low loss of the drug into the aqueous phase and the satisfactory encapsulation values. In addition, using dichloromethane as major solvent in the microencapsulation procedure is advantageous compared to other organic solvents as Chloroform or Benzene. Dichloromethane has a high solubility in water which allows fast mass-transfer between the dispersed and continuous phases. This leads to fast precipitation of the polymer and good drug entrapment ${ }^{15}$.
Regarding drug loading values, as more drug quantity is introduced, it is being more included into the polymeric microparticles. This explains the increasing \%DL from 0.2 to $1 \%$ as the drug mass increases from $10 \mathrm{mg}$ to 75 mg. In a previous study, the antifungal drug AmB was encapsulated into poly (DL-lactide-co-caprolactone) microspheres under the same conditions. Results showed lower drug loading compared to PCL. This could be explained by the more hydrophobic character of the polymer which enables it to retain more drug quantity 16 .

Table 1: \%DE, \%DL and particle size of the prepared formulations

\begin{tabular}{|c|c|c|c|c|}
\hline Formulation code & Drug Introduced $(\mathbf{m g})$ & \%DE & \%DL & size $(\boldsymbol{\mu m})$ \\
\hline A1 & 10 & 55 & 0.22 & 114 \\
\hline A2 & 20 & 61 & 0.48 & 115 \\
\hline A3 & 35 & 46 & 0.63 & 110 \\
\hline A4 & 50 & 36 & 0.71 & 126 \\
\hline A5 & 75 & 31 & 1.08 & 126 \\
\hline A6 & 100 & 28 & 1.13 & 123 \\
\hline
\end{tabular}

\section{In Vitro Release:}

Conforming to the low degradation and crystallinity of PCL among known polyesters used in targeted delivery, water penetration into PCL polymeric matrices is lower. This leads to drug release by diffusion and not by polymer degradation ${ }^{17}$.

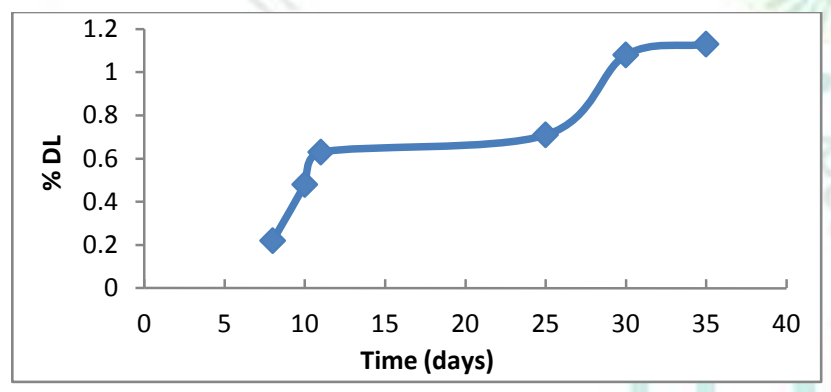

Figure 2: Variation of the release time according to $\% \mathrm{DL}$ of AmB-loaded formulations

Figure 2 shows the release time of the different $\% \mathrm{DL}$ of the prepared formulations. The release rate becomes slower at higher \%DL to reach a maximum of one month for formulation A5. In fact, at higher loadings, the chances of drug particles inside the polymer matrix to contact each other are high. This causes the formation of aggregates that need more time to dissolve, and thus more time for the drug to be released ${ }^{18}$.

Figure 3 presents the drug release profile of the available drug formulations. It is characterized by a burst release between days 5 and 10 where $30-40 \%$ of $\mathrm{AmB}$ was released. This is shown in particular for formulations with lower \% DL. The initial burst could be due to the dissolution of drug molecules that are present at the surface of microspheres ${ }^{14,19}$.

In opposite, an extended prolonged release was shown for the other formulations and required one month to go to completion. Since AmB is a hydrophobic drug, it is retained inside the core of the polymeric matrix after encapsulation not near the surface of particles as in the case of hydrophilic drugs. As a result, it requires more time to diffuse to the aqueous buffer medium and to be released ${ }^{8-10,15}$.

Besides, the polymer PCL is more hydrophobic than other polyesters. This contributes in making its degradation very slow in aqueous medium, and in turn suitable for controlled and extended drug release $9,10,14-$ 17.

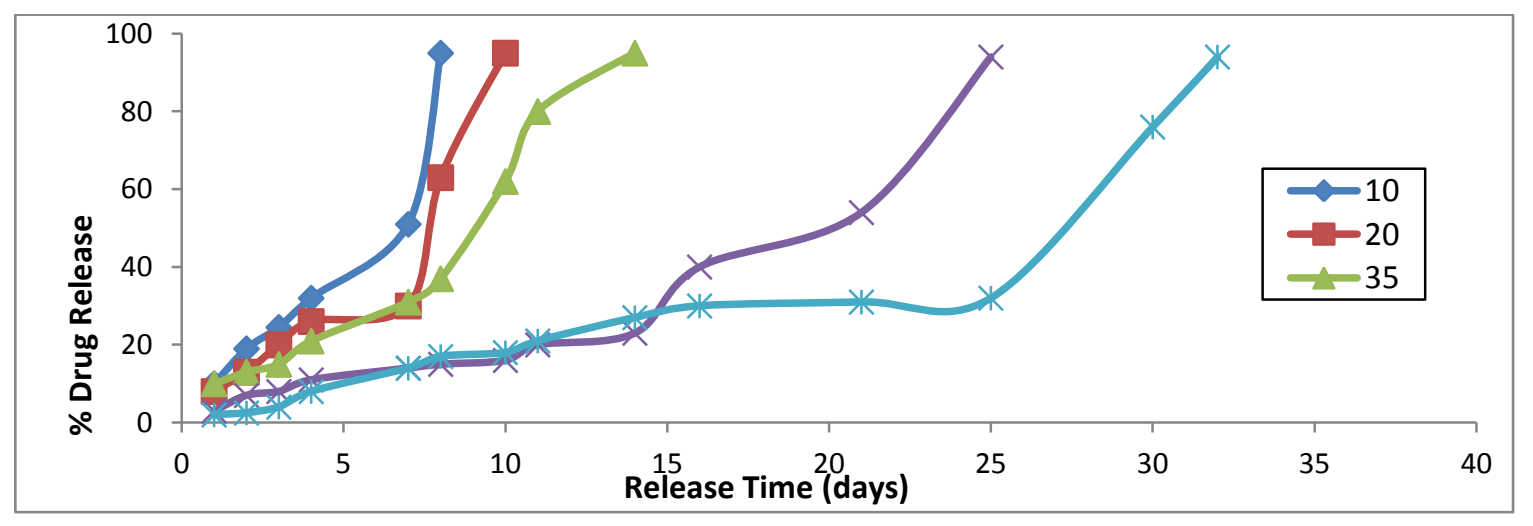

Figure 3: Drug release profile of the prepared AmB-loaded formulations 
FT-IR Analysis:

Table 2: Characteristics $\underset{20,21}{\text { Peaks of } \mathrm{AmB} \text { and PCL }}$ 4, 16,

\begin{tabular}{|c|c|c|}
\hline \multirow{4}{*}{ AmB } & Band $\left(\mathrm{cm}^{-1}\right)$ & Type of bond \\
\cline { 2 - 3 } & 1560 & $-\mathrm{C}=\mathrm{C}$ alkene \\
\cline { 2 - 3 } & $2900-3000$ & $-\mathrm{C}-\mathrm{H}$ stretching alkane \\
\cline { 2 - 3 } & $3300-3400$ & $\begin{array}{l}-\mathrm{OH} \text { alcohol } \\
-\mathrm{NH}_{2} \text { amine }\end{array}$ \\
\hline \multirow{4}{*}{ PCL } & 1160 & $-\mathrm{C}-\mathrm{O}$ ester \\
\cline { 2 - 3 } & 1365 & $-\mathrm{C}-\mathrm{H}$ banding alkane \\
\cline { 2 - 3 } & 1720 & $-\mathrm{C}=$ O Ketone \\
\cline { 2 - 3 } & $2900-3000$ & $-\mathrm{C}-\mathrm{H}$ bending alkane \\
\hline
\end{tabular}

FT-IR study was carried out in order to identify the purity and chemical stability of drug and polymer following the microencapsulation process. Table 2 shows the characteristic peaks of AmB and PCL.

Figures 3, 4 and 5 illustrate the spectra recorded on the IR spectrometer. Comparison of bands among the three spectra showed no significant large shift or deviation in the spectra of the drug and polymer when formulated into microspheres. All the characteristic peaks of AmB and PCL are showing in the spectrum of AmB-loaded microspheres (Table 2). These results indicate that the solvent evaporation was efficient in encapsulating the drug into PCL microspheres while remaining stable and intact.

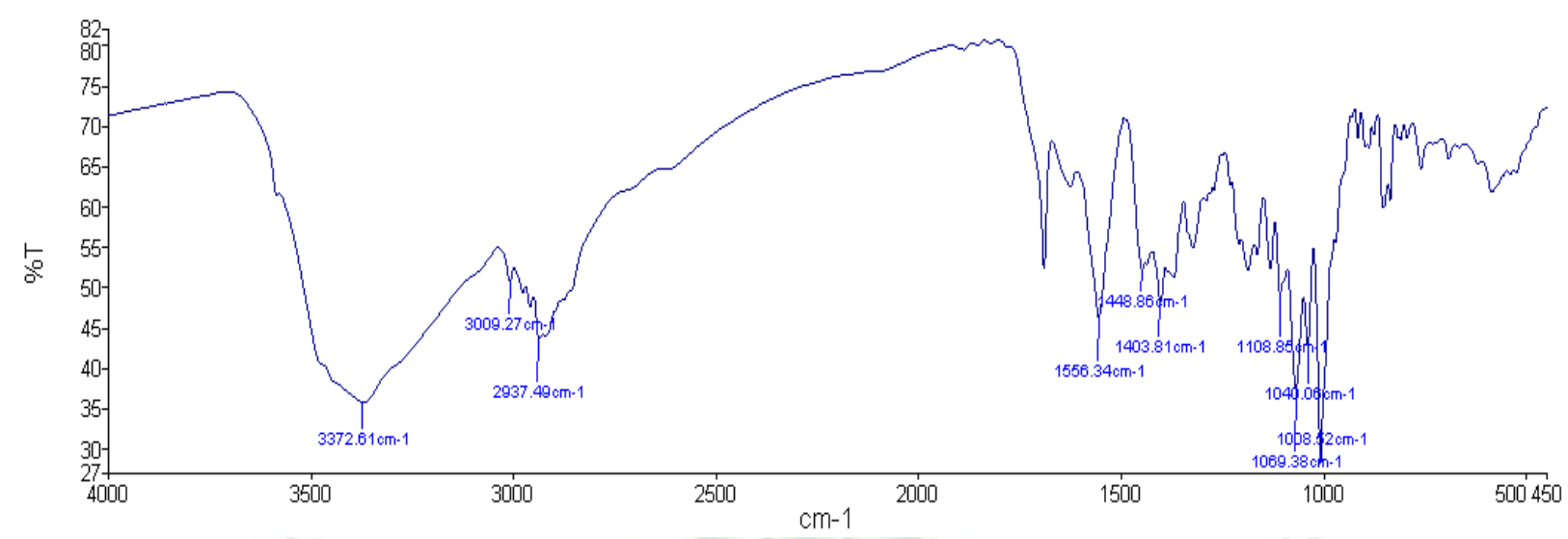

Figure 4: FT-IR spectrum of AmB

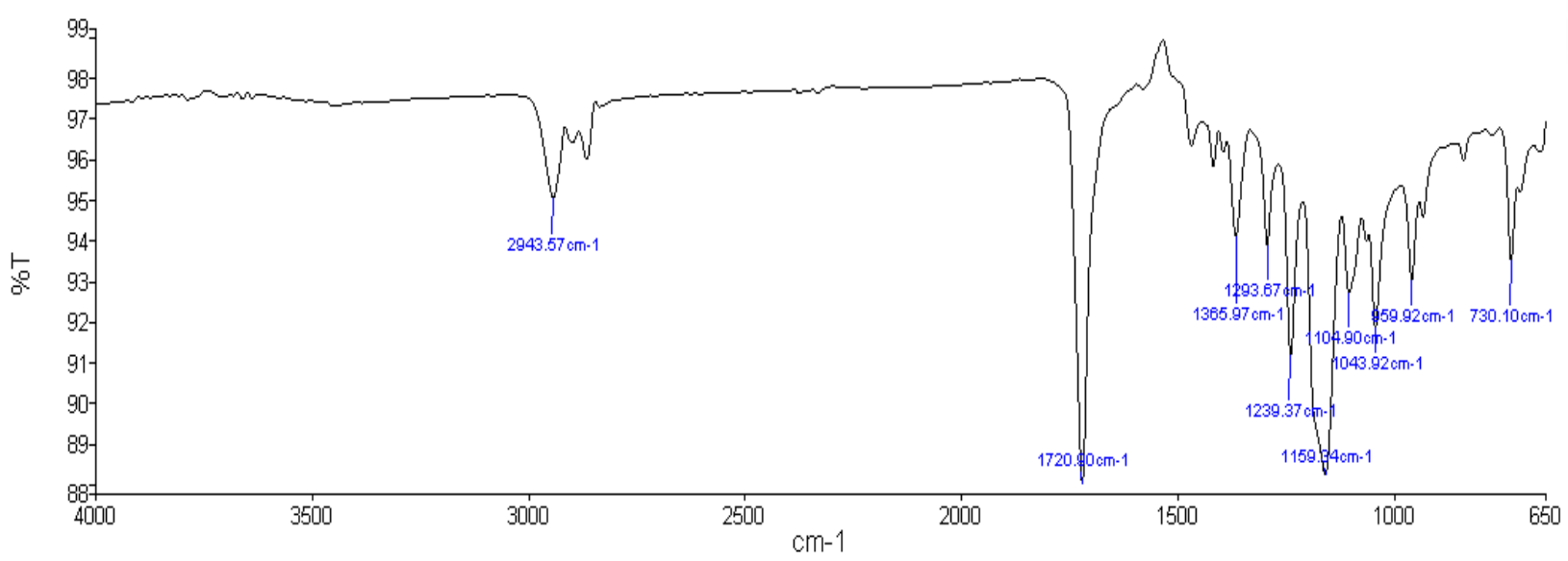

Figure 5: FT-IR spectrum of PCL blank microspheres

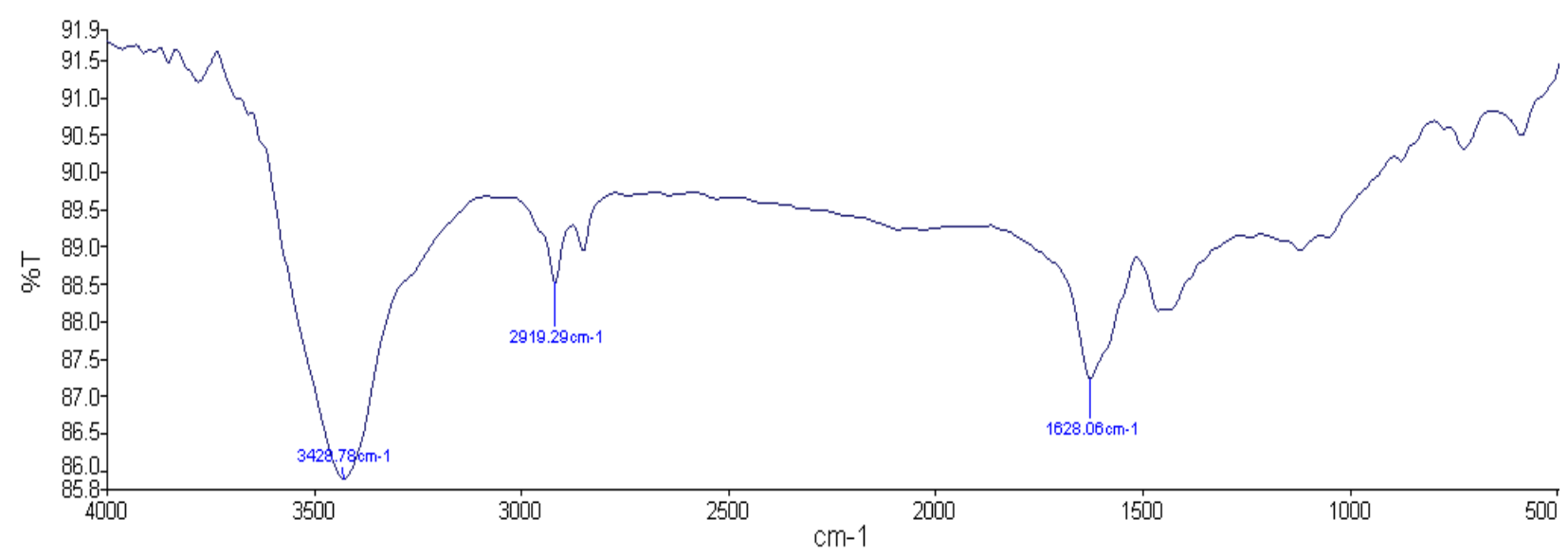

Figure 6: FT-IR spectrum of AmB-loaded microspheres 


\section{CONCLUSION}

In this project, the biodegradable polymer Polycaprolactone was used for the encapsulation of the antifungal agent Amphotericin B into porous and spherical particles with homogeneous sizes. Several samples were prepared by fixing the polymer quantity and modifying the drug mass. Drug encapsulation and loading percentages were reasonable, ranging between 28 and $61 \%$ and 0.22 and $1.13 \%$ respectively. The

\section{REFERENCES}

1- Dash TK, Konkimalla VB, Poly- $\epsilon$-caprolactone based formulations for drug delivery and tissue engineering: a review, Journal of Controlled Release, 2012; 158(1):15-33.

2- Yammine P, Maarawi T, Moussa D, Abdel-Massih R, Kassab $\mathrm{R}$, Effects of different surfactants on Indomethacin microspheres formulations, Journal of Advances in Chemistry, 2015; 11(4):3453-3462.

3- Ferreira SI, Bettencourt AF, Gonçalves MD, Kasper S, Bétrisey B, Kikhney J, Moter A, Trampuz A, Almeida AJ, Activity of Daptomycin- and Vancomycin-loaded polyepsilon-caprolactone microparticles against mature staphylococcal biofilms, International Journal of Nanomedicine, 2015; 10(1):451-4366.

4- Natarajan V, Krithica N, Madhan B, Sehgal PK, Formulation and evaluation of Quercitin polycaprolactone microspheres for the treatment of rheumatoid arthritis, Journal of Pharmaceutical Sciences, 2011; 100(1):195-205.

5- Woodruff MA, Hutmacher DW, The return of a forgotten polymer- polycaprolactone in the $21^{\text {st }}$ century, Progress in Polymer Science, 2010; 35(10):1217-1256.

6- Miladi K, Ibraheem D, Iqbal M, Sfar S, Fessi H, Elaissari A, Particles from preformed polymers as carriers for drug delivery, EXCLI Journal, 2014; 13:28-57.

7- Dhanaraju M, Sathyyamoorthy N, Sundar V, Preparation of polycaprolactone microspheres containing Etoposide by solvent evaporation method, Asian Journal of Pharmaceutical Science, 2010; 5(3):114-122.

8- Behara AK, Barik BB, Joshi S, Polycaprolactone based microspheres and nanospheres: a review, FS Journal of Pharmacy Research, 2012; 1(2):38-45.

9- Aydin O, Aydin B, Tezcaner A, Keskin D, Study of physicochemical structure and in vitro release behaviors of Doxycycline-loaded PCL microspheres, Journal of Applied Polymer Science, 2015; 132(14):41768 (1-13).

10- Mondal D, Griffith M, Venkatraman S, Polycaprolactonebased biomaterials for tissue engineering and drug delivery: current scenario and challenges, International Journal of Polymeric Materials and Polymeric Biomaterials, 2016; 65(5):255-265.

11- Aminu N, Baboota S, Pramod K, Singh M, Dang S, Ansari SH, Sahni JK, Ali J, Development and evaluation of Triclosan loaded poly- $\varepsilon$-caprolactone nanoparticulate system for the release rate was slow for the most of the formulations, and was directly affected by the degradation rate of the polymer and hydrophobicity of both drug and polymer. Finally, the drug-polymer compatibility study by FT-IR revealed their stability with no change in any chemical composition.

\section{Conflict of Interest}

All contributing authors declare no conflicts of interest.

treatment of periodontal infections, Journal of Nanoparticle Research, 2013; 15(11):1-15

12- Wang X, Wang Y, Wei K, Zhao N, Zhang S, Chen J, Drug distribution within poly( $\varepsilon$-caprolactone) microspheres and in vitro release, Journal of Materials Processing Technology, 2009; 209(1):348-354.

13- Dash T, Koncimalla V, Polymeric modification and its implication in drug delivery: poly( $\varepsilon$-caprolactone as a model polymer, Molecular Pharmaceutics, 2012; 9(9):2365-2379.

14- Waknis V, Jonnalagadda S, Novel poly-dl-lactidepolycaprolactone copolymer based flexible drug delivery system for sustained release of Ciprofloxacin, Drug Delivery, 2011; 18(4):236-245

15- Jelvehgari M, Valizadi H, Rezapour M, Nokhodchi A, Control of encapsulation efficiency in polymeric microparticle system of Tolmetin, Pharmaceutical Development and Technology, 2010; 15(1):71-79.

16- Yammine P, Kassab R, Moussa D, Moussa R, Poly(DLlactide-co-caprolactone) as drug carrier for antifungal drug Amphotericin B, 2012; 4(4):477-483.

17- Pepic D, Nikolic MS, Grujic S, Lausevic M, Djonlagic J, Release behaviour of Carbamazepine-loaded poly $(\varepsilon-$ caprolactone)/ poly(ethylene oxide) microspheres, Journal of Microencapsulation, 2013; 30(2):151-160.

18- Kassab R, Yammine P, Moussa D, Safi N, A comparative study of Doxycycline and Tetracycline polymeric microspheres, International Journal of Pharmaceutical Sciences and Research, 2014; 5(6):2452-2457.

19- Iqbal S, Rashid MH, Arbab AS, Khan M, Encapsulation of anticancer drugs (5-Fluorouracil and Paclitaxel) into polycaprolactone nanofibers and in vitro testing for sustained and targeted therapy, Journal of Biomedical Nanotechnology, 2017; 13(4):355-366

20- Angra P, Oettinger C, Pai SB, D’Souza MJ, Amphotericin B microspheres: a therapeutic approach to minimize toxicity while maintaining antifungal efficacy, Journal of Microencapsulation, 2009; 26(7):580-587.

21- Das S, Suresh PK, Nanosuspension: a new vehicle for the improvement of the delivery of drugs to the ocular surface: application to Amphotericin B, Nanomedicine: Nanotechnology, Biology, and Medicine, 2011; 7(2):242-247. 